\title{
Impacto del dolor en la incapacidad laboral. Metodología de valoración. Grados funcionales de limitación
}

\author{
Impact of pain on disability. Valuation methodology. Degrees of functional limitation
}

\author{
José Manuel Vicente Pardo ${ }^{1}$ \\ 1. Jefe Unidad Médica Equipo Valoración Incapacidades Gipuzkoa. INSS. \\ Recibido: 09-01-14 \\ Aceptado: 11-03-14 \\ Correspondencia \\ José Manuel Vicente Pardo \\ C/ Avenida Madrid 15, 3. D \\ San Sebastián 20011 \\ Gipuzkoa. España \\ jose-manuel.vicente@seg-social.es
}

Resumen

El abordaje de la valoración del dolor como incapacitante laboral es extremadamente difícil por la necesidad de objetivar y dimensionar las limitaciones derivadas del dolor y por la necesidad de ponerlas en relación con las capacidades requeridas por el trabajo. Así mismo valorar la servidumbre terapéutica o el impacto que pueda ocasionar la analgesia en el desarrollo de su trabajo. Cuestión más complicada es diferenciar la exageración o el sobredimensionamiento clínico del paciente, y más aún el componente simulador. No se puede hablar de dolor en singular, pues existen múltiples tipos de dolores y además cada uno de ellos se manifiesta de forma diferente, dependiendo de las características y condiciones del proceso causal y del propio sujeto. Este trabajo pretende exponer una metodología de trabajo en la consulta evaluadora de la capacidad laboral, unas consideraciones respecto de la posible simulación del dolor, un protocolo de actuación y finalmente una gradación de las limitaciones que puedan servir de ayuda en el complejo cometido de valorar la incapacidad laboral del dolor como un componente añadido a las limitaciones funcionales derivadas de su causa.

Material y método: Se han revisado hasta octubre de 2013 las siguientes bases de datos bibliográficas: SciELO, y PUBMED. Así como la Bibliografía y documentos de consulta citados en la misma.

Conclusiones: Sólo una correcta metodología en el reconocimiento médico de la valoración de la capacidad laboral y una referencia directa a su gradación limitante puede llevarnos a un adecuado juicio clínico laboral que elimine en parte el componente subjetivo del dolor y concluya en la objetivación del daño resultante en su capacidad laboral y en las capacidades perdidas que puedan ser exigidas en el trabajo. Este trabajo aporta las consideraciones para aminorar los problemas en la toma de decisiones en valoración médica de la capacidad laboral y una mayor concreción de las limitaciones y conclusiones en los informes médicos de evaluación.

Med Segur Trab (Internet) 2014; 60 (234) 133-142

Palabras clave: dolor, incapacidad laboral, metodología de valoración.

Abstract

The approach to the assessment of pain and crippling labor is extremely difficult by the need to objectify and measure the pain and limitations imposed by the need to relate them to the skills required for the job. Also evaluate the therapeutic servitude or the impact it may cause analgesia in the development of their work. More complicated issue is to differentiate clinical exaggeration or oversizing of the patient, and 
even more the simulator component. You can not speak of pain in the singular, as there are many types of pain and also each manifests differently depending on the characteristics and conditions of the causal process and the subject itself. This work aims to present a methodology for evaluating work in consulting work capacity, some considerations regarding the possible simulation of pain, a protocol and finally a gradation of the limitations that may assist in the complex task of assessing the pain disability as a component added to the resulting functional limitations of their cause.

Conclusions: Only a correct methodology in the medical assessment of work capacity and a direct reference to limiting gradation can lead to an adequate labor clinical judgment in part to eliminate the subjective component of pain and completed the objectification of the resulting damage in their ability to work and lost abilities that may be required at work. This paper provides considerations to mitigate the problems in decision-making in medical assessment of work capacity and greater realization of the limitations and conclusions in medical evaluation reports.

Med Segur Trab (Internet) 2014; 60 (234) 133-142

Keywords: pain, disability, valuation methodology. 


\section{INTRODUCCIÓN}

Dolor e incapacidad son situaciones que pueden presentarse asociadas pero que cuando se precisa valorar el carácter incapacitante laboral del dolor, es decir cuando debemos evaluar, objetivar y cuantificar la significación incapacitante laboral del dolor resulta tremendamente difícil por el componente subjetivo del dolor en su dimensionamiento.

Para valorar el dolor lo primero que debemos hacer es escuchar al paciente, valorar como expresa su intensidad, su localización, su disconfort, su repercusión en la autonomía funcional en las tareas personales, de relación y laborales, su repercusión psicológica la respuesta a los analgésicos y tipo de analgesia que precisa, su permanencia en el tiempo, pero también debemos cotejar la tolerancia al dolor, el umbral del mismo, la adaptación conductual, y la servidumbre terapéutica.

Si la valoración del dolor en responsabilidad civil, poner precio al dolor, (el quantum doloris) no queda resuelta su tasación, y su cuantificación es un mero artificio, pues el sufrir dolor no tiene un justiprecio compensador, valorar la incapacidad laboral que no es sino estimar la procedencia o no de una prestación económica resulta igualmente una entelequia, donde convergen intereses contrapuestos, por una parte el trabajador que sufre el dolor, de otra la compensación económica derivada de su impacto incapacitante.

Aún y con todo pretendemos establecer una metodología de trabajo que sirva de ayuda para una mejor toma de decisiones en valoración.

Situación Incapacitante Laboral:

Proceso patológico que genera un impedimento o una imposibilidad o incapacidad sobrevenida genérica, para trabajar, o para seguir ejecutando el contrato de trabajo que puede ser Temporal o Permanente.

Para que estemos ante una situación de incapacidad laboral deben de darse estos dos condicionantes:

1. Lesiones de tal entidad que causen Limitación y restricciones Funcionales intensas.

2. Limitaciones que puestas en relación con trabajo causan impedimento para el Trabajo o Imposibilidad Laboral.

Valorar una situación de Incapacidad Laboral es poner en relación las capacidades perdidas o las que mantiene el trabajador con las capacidades requeridas por el trabajo, las capacidades que precisa el trabajo.

Valoración de la Capacidad Laboral supone:

1. Determinar la existencia de una lesión o proceso patológico. Determinar las Deficiencias Objetivar sus consecuencias que son las Limitaciones orgánicas y/o Funcionales que origina en el trabajador.

2. Conocer las Tareas realizadas por el trabajador. Conocer los Requerimientos Profesionales y circunstancias específicas del ambiente laboral.

3. Establecer la relación entre las Limitaciones del Trabajador y los Requerimientos del Trabajo.

4. Concluir si está o no incapacitado.

Para valorar el dolor incapacitante tenemos que establecer:

1. Tipo de dolor.

2. Determinar el Manejo del dolor.

3. Objetivar su Limitación Funcional.

4. Identificar las Actividades Laborales o de la Vida Diaria que se ven limitadas o comprometidas a causa de la intensidad del dolor o por servidumbre terapeútica.

Impacto del dolor en la incapacidad laboral. 
El Dolor puede ser incapacitante, tanto en su presentación aguda que dará lugar al Inicio de una Incapacidad Temporal, como en la continuidad de la IT, y como consideración Incapacitante Permanente cuando se consolida como una residual secuelar estable y continuando con impedimento laboral precisa de su oportuna valoración en EVI.

El dolor es la principal causa de demanda de IT, como dolor agudo de cualquier clase en espera de diagnóstico y de necesidad de tratamiento, que impida trabajar.

En procesos de IT más de 35 días el dolor se presenta en un $35 \%$ de los casos, y en un $14 \%$ por dolor musculoesquelético (Lumbalgia $9 \%$, Cervicalgia $2 \%$, Hombro doloroso $2 \%$, Dolor en piernas 1\%). Siendo estos diagnósticos de los 15 primeros diagnósticos causales de IT.

Entre las causas de dolor incapacitante en su inicio están:

- Incertidumbre vital.

- Deterioro de salud.

- Fracaso de la prevención laboral.

- Inadecuada prevención sanitaria.

- El trabajo como causa de dolor incapacitante.

- El sobredimensionamiento clínico IT IP refugio.

Entre las causas de dolor incapacitante en su permanencia están:

- Incertidumbre vital.

- Deterioro de la salud derivado del proceso que genera el dolor.

- Inadecuada respuesta sanitaria.

- El sobredimensionamiento clínico la IT IP refugio.

Factores de riesgo de cronicidad del dolor (banderas amarillas):

- Individuales (Nivel educativo bajo, Niveles altos de dolor y discapacidad).

- Psicosociales (Angustia, Distrés, Humor depresivo, Somatización).

- Ocupacionales (Insatisfacción laboral, No disponibilidad de tareas ligeras al volver al trabajo, Trabajos de corte físico intenso).

El dolor como causa de IT prolongada, igual o superior a 12 meses.

El dolor crónico supone el 45 \% de estos supuestos. La mayoría de estos casos añaden un cuadro psíquico adaptativo.

Los dolores musculoesqueléticos sin organicidad, afectan a trabajadores del la administración pública, limpieza y hostelería en mayor proporción que a sectores industriales de riesgos físicos más intensos.

En un porcentaje elevado en estas situaciones queda por concluir el diagnóstico pues se está pendiente de pruebas exploratorias, o de tratamiento.

Muchos de estos pacientes son "hiperfrecuentadores" tanto en demanda de consultas en Atención Primaria, como en reiterados procesos de IT, y son "hiperconsumidores de recetas".

\section{VALORACIÓN DEL DOLOR INCAPACITANTE}

El dolor es una referencia del paciente, que puede constituir una enfermedad por sí misma, si no hay remedio terapéutico para su causa o sus manifestaciones.

El dolor tiene un marcado componente subjetivo y no siempre guarda relación con la severidad de la lesión. 
El dolor supone una merma importante de la calidad de vida del paciente, una pérdida de la satisfacción personal del paciente, un deterioro de su entorno inmediato y un coste y repercusión laboral muy altos.

\section{¿Cómo medir lo subjetivo? La perspectiva distorsionante del refiere}

Nuestro paciente es un ser sufriente.

Con impedimento para el trabajo por dolencias en espera de mejora con tratamiento.

Con limitaciones que impiden trabajar y trabajo con tareas que están contraindicadas con su enfermedad.

Nuestro paciente trabajador, en el momento de valoración No busca un diagnóstico, ni un tratamiento, Sino la justificación de una situación con una compensación económica.

La consecuencia de la valoración será un subsidio.

Busca, pretende o disfruta de una prestación económica, por pérdida de salud que impide trabajar

Tal vez pueda o no trabajar, tal vez deba o no trabajar, tal vez quiera o no trabajar, pero también sabemos que gana la guerra del quiere al puede o al debe trabajar.

Nuestro paciente:

- Experimenta

- Expresa

- Expone

- Explica sus síntomas.

Pero se complica su valoración cuando:

- Exhibe

- Exacerba

- Exagera

- Expande

- Extiende

- Explota

- Exprime

- Escenifica sus síntomas.

Y entonces, se acrecienta la dificultad para objetivar, y se da el fenómeno no infrecuente del sobredimensionamiento de la clínica.

Nuestra labor como médicos evaluadores en el INSS se trata mediante el informe médico de valoración establecer un juicio clínico laboral sobre la justa correspondencia de la situación presente o alegada.

Evaluar es identificar, que es objetivar, medir que es establecer la intensidad y concluir que no es sino establecer un juicio clínico laboral.

\section{Dolor. Simulación o Sobredimensionamiento clínico, Signos de Vicente:}

- Actitud teatralizante en la consulta desde su presentación.

- Exhibición aparatosa de síntomas.

- Ganancia de enfermedad. 
- Quejas poco consistentes, inoperancia de cuantos tratamientos ha seguido, con nula mejoría.

- Persistencia de síntomas más allá de la evolución esperada.

- Nula o escasa organicidad del proceso y abundante componente funcional exploratorio.

- Respuesta dolorosa a cualquier requerimiento mínimo exploratorio, "totalgia", incluyendo expresiones de respuesta a pruebas que nada tienen que ver con el cuadro clínico simulado.

- Diferencia de hallazgos exploratorios en las maniobras directas, con conciencia del enfermo, y en las maniobras diferidas. Exploración paradójica.

- Inconsistencia de síntomas o diferencias notables con respecto a consultas efectuadas por otros compañeros del ámbito asistencial y las efectuadas para valoración del daño.

- En su exageración da peores resultados que el azar en los test, toda prueba le estimula sus síntomas e incluso tiene más síntomas que los que corresponden al proceso que simula.

- "Síndrome de Lección Aprendida", el paciente conoce los síntomas y signos de su proceso y los adelanta antes de proceder a la maniobras de exploración.

- Los síntomas desaparecen fuera del ámbito de la valoración, cesan al no sentirse observados por el médico evaluador.

- Cesan al obtener su objetivo en litigio, aunque se reproducirán caso de revisión.

- No acepta su curación, si así fuera perdería sus beneficios obtenidos.

- No colabora, miente, distorsiona o exagera. Manipula informes, oculta aquellos que no le benefician y pondrá pegas ante examen de terceros o realización de pruebas funcionales.

\section{Propuesta para resolver esta dificultad: Establecer una metodología en valo- ración del dolor}

No existe ninguna prueba de carácter objetivo que permita medir la intensidad del dolor que padece una persona, y su carácter incapacitante.

¿Podemos afirmar que el dolor es un signo suficiente para evaluar una Incapacidad Laboral?

- Sí, pero No por sí solo.

- Si cuando se presenta asociado a la disfunción que causa, o asumido como parte integrante y esperable de los síntomas de un proceso.

El dolor en Incapacidad Temporal puede presentarse por sí sólo aunque no hallemos lesión, de hecho es motivo de las principales causas de baja (lumbalgia, hombro dolorosos y cervicalgia).

El dolor en Incapacidad Permanente, si proviene de baja larga, suele acompañar a lesión objetivada y en ocasiones se presenta con comorbilidad o pluriafectación lesional.

\section{Metodología de valoración del dolor incapacitante. Protocolo de actuación}

Debemos acudir a la apreciación de elementos de carácter objetivo.

- HISTORIA CLÍNICA. Anamnesis correcta.

- EXPLORACIÓN FÍSICA. Exploración Funcional Específica.

- PRUEBAS. Pruebas Exploratorias Complementarias.

- TRATAMIENTO. Valoración Terapéutica.

Ante el Dolor incapacitante debemos de preguntarnos:

1. ¿Cuál es la causa del dolor?

2. ¿Qué Tratamiento ha seguido y con qué respuesta? 
3. ¿Qué Limitaciones Funcionales causa?

4. ¿Cómo cuenta el paciente es su dolor?

5. ¿Cuál es la servidumbre terapéutica por la analgesia que precisa?

6. ¿Qué capacidades están limitadas o mermadas, qué capacidades mantiene?

7. ¿Qué capacidades requiere su trabajo?

Si el dolor es un intangible, ¿cómo pretender medir lo que no es medible?

El dolor tiene un marcado componente subjetivo, y además el dolor no siempre guarda relación directa con la severidad de la lesión que lo causa.

- Los métodos subjetivos mediante cuestionarios o escalas tienen el sesgo de cómo se siente el paciente o como siente el dolor, no es una cuantificación de cuánto dolor existe. Tanto con las escalas unidimensionales como multidimensionales.

- Los métodos objetivos, suponen un acercamiento más significativo ya que valoran el comportamiento psicológico, los diferentes cambios fisiológicos que el dolor comporta, que son medibles, así como, las determinaciones bioquímicas que se producen, si bien estas son de escasa consistencia, poca sensibilidad poca especificidad.

\section{Pautas de actuación para valorar el dolor}

- Recoger información. Saber Escuchar. Saber Preguntar.

- Valorar respuesta al tratamiento y tipo de analgesia.

- Observar al paciente.

- Valorar las pruebas complementarias. Los hallazgos en pruebas funcionales complementarias.

- Exploración neurológica y musculoesquelética. Efectuar Maniobras diferidas, combinadas y de distracción. Y en el dolor musculoesquelético sin organicidad buscar Signos Waddellianos.

\section{Metodología de valoración. Grados funcionales 0/4}

Es necesario efectuar una Gradación Funcional de las limitaciones, porque no basta decir (describir) que existe una limitación es necesario establecer su magnitud, su alcance, su intensidad (cuantificar)

Los Grados Funcionales, son criterios médicos de valoración atribuibles a situación, estableciendo 5 grupos funcionales que reúnen grados del 0 al 4 según su menor o mayor severidad limitante.

\section{Grado Funcional 0}

- Dolor leve, soportable que no precisa de tratamiento.

- Plena capacidad funcional cotidiana.

\section{Grado Funcional 1}

- Dolor agudo, que responde a tratamiento aunque este sea intenso. (ascensor analgésico, puede precisar opiáceos débiles, luego cede con paso a escalón $1 .^{\circ}$

- Dolor crónico insidioso, que precisa ocasionalmente analgésicos de $1 .^{\circ}$ escalón, con buena respuesta o dolor leve continuado.

- Impedimento funcional temporal en espera de respuesta terapéutica, según la intensidad con capacidades funcionales limitadas para aquellas actividades $o$ despliegue funcional que acentúen el mismo con funcionalidad respetada para otras. 


\section{Grado Funcional 2}

- Dolor moderado, penoso, persistente con respuesta sólo parcial al tratamiento, precisa analgésicos de $2 .^{\circ}$ escalón.

- Dolor que se intensifica a la movilización.

- Puede suponer una limitación funcional para el despliegue de actividades de corte físico moderado o exigente o merma de sensorio o capacidad de respuesta o de alarma.

\section{Grado Funcional 3}

- Dolor moderado fuerte, exasperante, persistente, a la actividad, sin respuesta eficaz al tratamiento, precisará analgésicos de $3 .^{\circ}$ escalón.

- Funcionalidad reducida para vida dinámica, y también limitación funcional para actividades sedentarias.

- Afectación psicológica acusada, puede interrumpir el sueño, y causar deterioro de la vida personal.

\section{Grado Funcional 4}

- Dolor insoportable que no responde a opioides mayores de $3 .^{\circ}$ escalón.

- Analgesia de $4 .^{\circ}$ escalón.

- Requiere analgesia con métodos invasivos.

- Dolor muy intenso en reposo.

- Signos vegetativos.

- Limitado para llevar una vida con autonomía funcional, deterioro psíquico, depresión manifiesta, pensamiento dificultoso, impedimento para reposo nocturno, dificultades para desplazamiento, encamado.

\section{Escalas de ayuda en la valoración disfuncional en lo cotidiano:}

Escala Thierry, Escala Karnofsky, Escala Ecog.

\section{ESCALA THIERRY}

\begin{tabular}{ll}
\hline DOLOR POCO IMPORTANTE & $\begin{array}{l}\text { No es síntoma fundamental, no produce incapacidad ni precisa tratamiento } \\
\text { Destacado como síntoma, produce incapacidad pero puede ser calmado } \\
\text { DOLOR MODERADO }\end{array}$ \\
DOLOR IMPORTANTE & $\begin{array}{l}\text { Produce incapacidad, no puede ser superado simplemente con analgésicos } \\
\text { Es muy importante por su intensidad, consecuencias y persistencia hasta el } \\
\text { punto que anula toda actividad del sujeto. } \\
\text { Precisa de analgesia de tercer escalón opiáceos o morfina o sedación } \\
\text { completa }\end{array}$ \\
\hline
\end{tabular}

\section{ESCALA DE VALORACIÓN FUNCIONAL DE KARNOFSKY}

\begin{aligned} & \hline 100: Normal, sin quejas, sin indicios de enfermedad. \\ & 90: Actividades normales, pero con signos y síntomas leves de enfermedad. \\ & 80: Actividad normal con esfuerzo, con algunos signos y síntomas de enfermedad. \\ & 70: Capaz de cuidarse, pero incapaz de llevar a término actividades normales o trabajo activo. \\ & 60: Requiere atención ocasional, pero puede cuidarse a sí mismo. \\ & 50: Requiere gran atención, incluso de tipo médico. Encamado menos del $50 \%$ del día. \\ & 40: Inválido, incapacitado, necesita cuidados y atenciones especiales. Encamado más del $50 \%$ del día. \\ & 30: Inválido grave, severamente incapacitado, tratamiento de soporte activo. \\ & 20: Encamado por completo, paciente muy grave, necesita hospitalización y tratamiento activo. \\ & 10: Moribundo. \\ & $0:$ Fallecido. \\ & \hline\end{aligned}


La Escala de Valoración Funcional de Karnofsky permite conocer la capacidad del paciente para poder realizar actividades cotidianas.

Es un elemento predictor independiente de mortalidad, tanto en patologías oncológicas y no oncológicas. Sirve para la toma de decisiones clínicas y valorar el impacto de un tratamiento y la progresión de la enfermedad del paciente. Un Karnofsky de 50 o inferior indica elevado riesgo de muerte durante los 6 meses siguientes.

\section{Escala ECOG}

La escala ECOG valora la evolución de las capacidades del paciente en su vida diaria manteniendo al máximo su autonomía. Este dato es muy importante cuando se plantea un tratamiento, ya que de esta escala dependerá el protocolo terapéutico y el pronóstico de la enfermedad. La escala ECOG se puntúa de 0 a 5 y sus valores son:

\begin{tabular}{ll}
\hline ECOG 0: & $\begin{array}{l}\text { El paciente se encuentra totalmente asintomático y es capaz de realizar un trabajo y } \\
\text { actividades normales de la vida diaria. }\end{array}$ \\
\hline ECOG 1: & $\begin{array}{l}\text { El paciente presenta síntomas que le impiden realizar trabajos arduos, aunque se } \\
\text { desempeña normalmente en sus actividades cotidianas y en trabajos ligeros. El paciente } \\
\text { sólo permanece en la cama durante las horas de sueño nocturno. }\end{array}$ \\
\hline ECOG 2: & $\begin{array}{l}\text { El paciente no es capaz de desempenar ningún trabajo, se encuentra con síntomas que le } \\
\text { obligan a permanecer en la cama durante varias horas al día, además de las de la noche, } \\
\text { pero que no superan el 50\% del día. El individuo satisface la mayoría de sus necesidades } \\
\\
\text { personales solo. }\end{array}$ \\
\hline ECOG 3: & $\begin{array}{l}\text { El paciente necesita estar encamado más de la mitad del día por la presencia de síntomas. } \\
\text { Necesita ayuda para la mayoría de las actividades de la vida diaria como por ejemplo el } \\
\text { vestirse. }\end{array}$ \\
\hline ECOG 4: & $\begin{array}{l}\text { El paciente permanece encamado el 100\% del día y necesita ayuda para todas las } \\
\text { actividades de la vida diaria, como por ejemplo la higiene corporal, la movilización en la } \\
\text { cama e incluso la alimentación. }\end{array}$ \\
\hline ECOG 5: & Paciente fallecido. \\
\hline
\end{tabular}

\section{REFERENCIAS BIBLIOGRÁFICAS}

1. Vicente Pardo José Manuel. Incapacidad laboral y dolor. Revista Medical Economics 23 de diciembre de 2011.

2. Vicente Pardo José Manuel. Ponencia XIV Congreso Internacional de Prevención de Riesgos Laborales. Toledo 2012. Impacto del dolor en la incapacidad laboral y metodología de valoración.

3. González Ordi H, Santamaría Fernández P. Adaptación española del Inventario Estructurado de Simulación de Síntomas - SIMS. Madrid: TEA Ediciones, 2009.

4. American Pain Society Quality of care Committee. Quality improvement guidelines for the treatment of acute pain and cancer pain. JAMA 1995.

5. Torrubia R, Baños JE. Evaluación del dolor. Tratamiento del dolor. Teoría y Práctica, 2002. 2. ${ }^{a}$ ed. Editorial Permanyer.

6. Besson JM. The neurobiology of pain. Lancet 1999

7. González Ordi H, Capilla Ramírez P, Matalobos Veiga B. Simulación del dolor en el contexto médico legal. Monográfico Dolor Crónico. Clínica y Salud 2008.

8. Jacobsen P, Kriegstein O, Portenoy R. Breakthrough pain: prevalence and characteristics. Proc Am Soc Oncol 1994.

9. Aliaga A, Baños JE, Barutell C, Molet J, Rodríguez de la Serna A, coordinadores. Tratamiento del dolor: teoría y práctica. Barcelona: Editorial MRC; 1995.

10. Capilla Ramírez P, González Ordi H. Protocolo para la detección de la simulación del dolor en la práctica clínica: estudio de casos. Revista Trauma vol. 20 suplemento 42009 Fundación Mapfre.

11. Tunks E, Bellisimo A. Coping with the coping concept: a brief comment. Pain. 1988.

12. Muriel Villoria C; Madrid Arias J. L.; Valoración del Dolor, estudio y tratamiento del dolor crónico. ELA 1994.

13. Torrubia R. Banños J. E.; Evaluación del Dolor. Teoría y práctica. MCR. SA. 1995. 
14. Miró J. Dolor crónico. Procedimiento de Evaluación e intervención psicológica. Desclée de Brower SA 2003.

15. Desari M. D. Taxonomía de los síndromes dolorosos, clasificación del dolor. EDIDE SL 2002.

16. Serrano-Atero, M S, Caballero, J; Cañas, A; García-Saura, P L; Serrano-Álvarez, C; Prieto, J Valoración del dolor. R e v. Soc. Esp. Dolor 9: 2002.

17. Alonso, J., Prieto, L., y Antó, J. M. (1995) La versión española del SF-36 Health Survey (Cuestionario de Salud SF-36): un instrumento para la medida de los resultados. Medicina Clínica.

18. Esteve, R., López, A. E. y Ramírez, C. (1999). Evaluación de estrategias de afrontamiento al dolor crónico. Revista de Psicología de la Salud.

19. Clínica y Salud v.19 n.3 Madrid dic.-ene. 2008. Evaluación psicológica del dolor.

20. Valoración de la Incapacidad J. A. Ojeda Gil.

21. Wilkie D, Savedra MC, Holzemer WL et al. Use of the McGill Pain Questionnaire to measure pain: a metaanalysis. Nursing Res 1990

22. Y. Escobar, M. Dómine, F. Valcárcel and J. Contreras. Evaluation of sensitivity to change of the MPAC scale, validated in Spanish, in pain measurement in cancer patients.

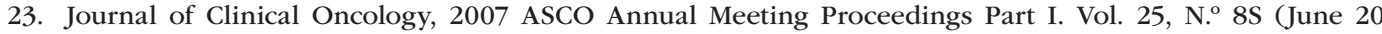
Supplement), 2007

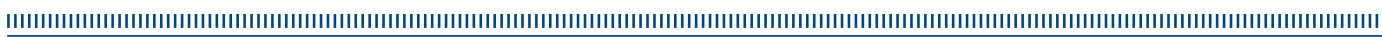

